\title{
App für Frühchen-Eltern
}

— Die Neonatologie der Klinik für Kinderund Jugendmedizin des Universitätsklinikums Carl Gustav Carus Dresden unterstützt die Entwicklung neuer Formen der Kommunikation bei der Betreuung von zu früh- oder krank geborener Kinder. Seit dem 1. Januar 2016 können Eltern von früh- oder krankgeborenen Kindern mit ihrem Smartphone die Software "Neo-App\#Tagebuch" nutzen. „Eltern, deren Kinder deutlich zu

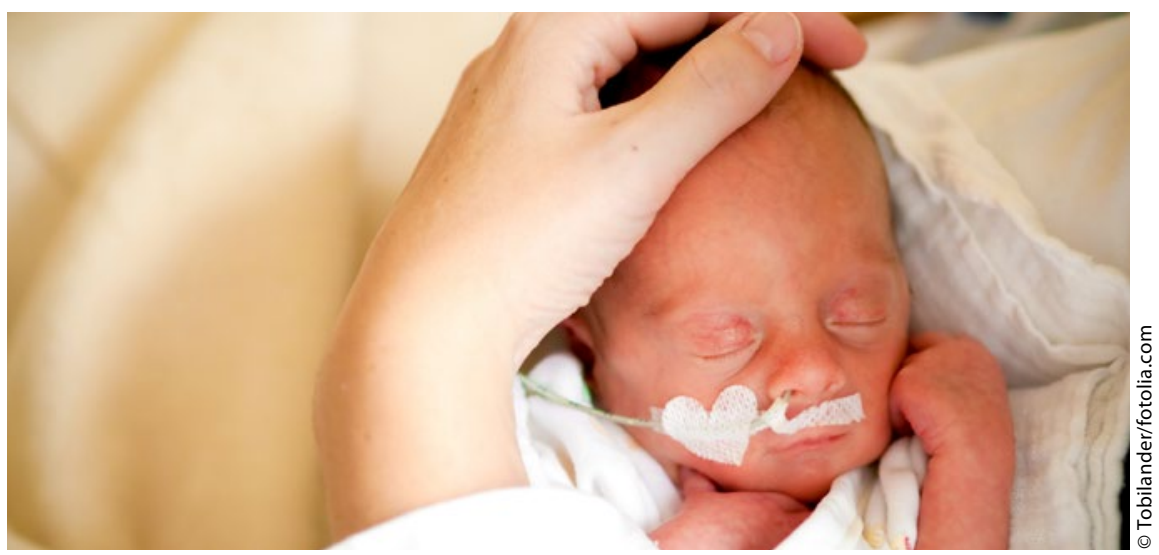

früh oder krank geboren werden und die in den ersten Monaten um das Überleben ihres Babys bangen, fühlen sich während der stationären Versorgung im Krankenhaus und in der Anfangszeit zu Hause im Umgang mit ihrem Kind mitunter noch nicht ausreichend sicher", erklärt der Psychologe in der Neonatologie des Uniklinikums Dresden, Privatdozent Dr. Jörg Reichert. Durch den Einsatz der Smartphone-Anwendung sollen betroffene Eltern einen einfacheren Überblick über die Entwicklung ihres Kindes bekommen und dadurch im Umgang mit ihrem Nachwuchs schnell an Sicherheit gewinnen. Später kann das App-Tagebuch für die Patienten und den behandelnden Arzt eine wichtige Erinnerungshilfe sein.

www.uniklinikum-dresden.de www.akili-innovation.com

\section{Mehr Sicherheit \\ Assistierte Pflege: Gut vernetzt}

— Vom intelligenten Pflegebett für die Prävention und Behandlung von Dekubitus über Beatmungspflege zuhause oder die entlastende Oberkörperorthese für körperlich anstrengende Pflegeabläufe bis hin zum Ortungs- und Monitoringsystem für den Notfall: Blicke in die Zukunft der Pflegepraxis erlaubte die Abschlussveranstaltung der Fördermaßnahme „Assistierte Pflege von morgen" des Bundesministeriums für Bildung und Forschung in Erlangen. Rund 400 Expertinnen und Experten aus ganz Deutschland informierten sich in der Heinrich-Lades-Halle anhand einer Ausstellung über die Ergebnisse von 17 geförderten Forschungsprojekten zur ambulanten technischen Unterstützung und Vernetzung von Patienten, Angehörigen und Pflegekräften.

Bedarfsorientierte Lösungen: Der Parlamentarische Staatssekretär im Bildungs-ministerium Stefan Müller betonte, dass Technik den Pflegenden nicht ersetzen, sondern inn und den Pflegebedürftigen -ausgerichtet an deren Bedürfnissen - unterstützen sowie mehr Zeit für menschliche Zuwendung schaffen solle. Als Ziele der 2012 gestarteten
Fördermaßnahme benannte er außerdem, "die Innovationsstärke Deutschlands in der Medizintechnik auf die Pflege auszuweiten" und "Deutschland als Leitanbieter für innovative Lösungen zu etablieren.“ Für den an zwei Projekten beteiligten Gastgeber, das Fraunhofer Institut für Integrierte Schaltungen IIS, erklärte der stellvertretende Institutsleiter Bernhard Grill, dass bei den Forschungen und Lösungen „Hilfe zur Selbsthilfe“ im Vordergrund stehe, technische, soziale und wirtschaftliche Gesichtspunkte vereint und alle relevanten Akteure eingebunden werden sollten.

Vier Themenfelder - 17 Projekte: Die in punkto Umsetzungsreife unterschiedlich weit gediehenen Projekte widmeten sich vier Themenfeldern: Der Sicherheit und dem Wohlbefinden von Pflegebedürftigen, der Unterstützung von Pflegenden, der Pflege in komplexen Situationen und der vernetzten Pflege im Quartier und in den Regionen. Geruchssensorik bei Inkontinenz, intelligente Trinkbecherhalter, interaktive Lichtsysteme oder "Smart-Items-Technologien" zur Unterstützung einer korrekten
Medikamenteneinnahme etwa können künftig den Alltag und die soziale Teilhabe Pflegebedürftiger erleichtern. Erkennertechnologien für den Notfall, sensorgestützte Personaleinsatz- und Tourenplanung oder eine Plattform für deren Vernetzung sollen darüber hinaus die Pflegekräfte entlasten.

Bei dementiellen Erkrankungen oder der Palliativpflege können Trainingskonzepte für die Betreuung kognitiv beeinträchtigter Senioren, aber auch Systeme für die soziale Interaktion alleinlebender Palliativpatienten und Lösungen für eine integrative ambulante Palliativversorgung Angehörige und Pflegekräfte unterstützen. Geht es schließlich um die lückenlose Versorgung von Pflegebedürftigen auch im ländlichen Raum, sollen intelligente Kommunikationstechnologien die Koordination der Akteure erleichtern.

Abschlussveranstaltung "Assistierte Pflege von morgen“; Erlangen, 10. Dezember 2015

(Veranstalter: Frauenhofer-Institut für Integrierte Schaltungen IIS) 